Article

\title{
A Pairing-Based Three-Party Authenticated Encryption Scheme without Shared Secrets
}

\author{
Han-Yu Lin (1) \\ Department of Computer Science and Engineering, National Taiwan Ocean University, Keelung 202, Taiwan; \\ hanyu@mail.ntou.edu.tw
}

Received: 5 April 2019; Accepted: 28 April 2019; Published: 1 May 2019

\begin{abstract}
The Traditional Authenticated Encryption (AE) scheme is a single-user cryptographic mechanism which only enables one designated verifier to authenticate the ciphertext. Although several group-oriented AE variants have also been proposed to eliminate such a limitation, they require shared verification. This motivated us to think of a scenario of three-party communication environments where each party runs independent processes without cooperation. In this paper, we realize a novel three-party AE (abbreviated to TPAE) scheme in which two designated verifiers can solely decrypt the same ciphertext and then inspect the validity of embedded signature. Additionally, we also show that our TPAE construction is computationally secure using the well-defined IND-CCA2 and the EF-CMA adversary games in the proof model of random oracles. The comparison results will demonstrate the computational efficiency of our mechanism.
\end{abstract}

Keywords: authenticated encryption; digital signature; three-party; bilinear pairings; shared secret

\section{Introduction}

In public key cryptosystems [1], digital signature schemes [2-4] are important mechanisms that serve the same function of handwritten signatures in the real world. A significant property of digital signatures is nonrepudiation [5], which guarantees that a signer cannot deny their generated signatures later.

To further provide the property of confidentiality [6,7] for some special signature applications such as online auction and electronic transactions, one can employ the so-called two-step measure which means an encryption process is performed after a signing operation. Nevertheless, this approach is inefficient.

In 1994, to provide a better solution, Horster et al. [8] introduced a hybrid method known as the authenticated encryption (AE) scheme that can fulfill the characteristic of confidentiality and authenticity [9]. In such an approach, a signer combines the intended recipient's public key with the signing operation. In this way, the resulted authenticated ciphertext must be verified by the person holding the knowledge of correct private key. Thus, an AE scheme no longer exhibits the characteristic of public verification due to the confidentiality concern. However, this trait also leads to another problem of later dispute provided that a signer denies his/her behavior of making the signature. A designated verifier will have a difficulty in proving the signer's dishonesty without revealing his private key information.

To handle the above issue, Araki et al. [10] addressed a signature mechanism of limited verifier and an optional arbitration procedure is supplied. Yet, the procedure requires the signer's assistance to announce an extra parameter. Once the signer refuses cooperation, the arbitration mechanism is useless. Moreover, Zhang and Kim [11] showed that an adversary can choose an arbitrary message to launch universal forgery attacks against Araki et al.'s work successfully. 
Later, $\mathrm{Wu}$ and $\mathrm{Hsu}$ [12] proposed a convertible AE protocol admitting a designated verifier to solely conduct the arbitration steps and then reveal a converted signature for public verification. A noticeable property of the $\mathrm{Wu}-\mathrm{Hsu}$ scheme is that the signature conversion steps are computation-free, i.e., an originally signed signature would be acquired within the ciphertext decryption procedure. Next, Huang and Chang [13] also presented another enhanced scheme. However, Lv et al. [14] specified that the semantic security is not satisfied in both the Huang-Chang and the Wu-Hsu schemes. Concretely speaking, an adversary can easily distinguish a given authenticated ciphertext from only two candidate messages.

In 2005, Yang [15] proposed a secure scheme with provable security. In 2008, Chien [16] came up with a new $\mathrm{AE}$ variant permitting either an original signer or a determined recipient to run the conversion steps of signature. Constructed from famous ElGamal cryptosystems, in 2009, Lee et al. [17] introduced the ElGamal-based variant. In the same year, Wu and Lin [18] showed how to use RSA cryptosystems to build a concrete AE construction with convertibility. Aim at reducing the impact caused by key-compromise attacks, Hsu and Lin [19] presented a key-insulated AE scheme in which a helper is able to renew users' short-term private keys periodically with the increment of time periods.

Consider group-oriented applications, in 2011, Hsu and Lin [20] presented a convertible variation of multi-AE (a.k.a. MAE) method. To provide more flexible signing policy, in 2014 Lin [21] further introduced a threshold-AE scheme. In 2017, a new AE scheme supporting multisigner and the functionality of proxy delegation was also introduced [22]. Up to present, lots of AE variants [23-31] have been proposed. However, all of existing mechanisms puts the emphasis on either the single-user setting or the group-oriented environments with cooperative groups. In this paper, we aim at proposing a better alternative for three-party communication environments where each entity usually runs independent processes without sharing their secrets with each other.

\section{Preliminaries}

Before presenting the proposed mechanism, we first revisit the operations of bilinear pairing together with some famous cryptographic assumptions, which will be utilized as the underlying building blocks of our work.

\section{Concept of Bilinear Pairing}

Let the symbols of $G_{1}$ and $G_{2}$ be an additive and a multiplicative group, respectively. Both groups have the same prime order $q$. A bilinear pairing $e$ is expressed as $e: G_{1} \times G_{1} \rightarrow G_{2}$. Some properties of bilinear pairing $e$ are described as follows:

(i) Bilinearity:

$$
e\left(Q_{1}+Q_{2}, W\right)=e\left(Q_{1}, W\right) e\left(Q_{2}, W\right)
$$$$
e\left(Q, W_{1}+W_{2}\right)=e\left(Q, W_{1}\right) e\left(Q, W_{2}\right)
$$

(ii) Nondegeneracy: We say that in the group $G_{2}$, the value $e(Q, Q)$ is regarded as a generator provided that, in the group $G_{1}, Q$ is also a generator.

(iii) Computability: There exists an efficient polynomial-time algorithm to compute $e(P, Q)$ for any $P$, $Q \in G_{1}$.

\section{Elliptic Curve Discrete Logarithm Problem and Assumption}

An Elliptic Curve Discrete Logarithm Problem (ECDLP) is to find some $x \in Z_{q}^{*}$ satisfying the equality of $X=x Q$, where $X$ and $Q$ are two known points over an elliptic curve $E$. The ECDL assumption asserts that it is almost negligible advantage $\varepsilon$ for any probabilistic algorithm $\mathcal{A}$ running in polynomial-time to solve ECDLPs. More precisely, the ECDL assumption is denoted by the following probability inequality:

$$
\operatorname{Pr}\left[\mathcal{A}(Q, x Q)=x ; x \leftarrow Z_{q}^{*},(Q, x Q) \leftarrow G_{1}^{2}\right] \leq \varepsilon .
$$




\section{Bilinear Diffie-Hellman Problem and Assumption}

A Bilinear Diffie-Hellman Problem (BDHP) was used to calculate $e(Q, Q)^{x y z} \in G_{2}$ from four given values $(Q, X, Y$, and $Z)$ of the group $G_{1}$. In particular, $X=x Q, Y=y Q$ and $Z=z Q$ for some $x, y, z \in Z_{q}^{*}$. The $\mathrm{BDH}$ assumption asserts that it is almost negligible advantage $\varepsilon$ for any probabilistic algorithm $\mathcal{A}$ running in polynomial-time to solve BDHPs. Precisely speaking, the BDH assumption is denoted by the following probability inequality.

$$
\operatorname{Pr}\left[\mathcal{A}(Q, x Q, y Q, z Q)=e(Q, Q)^{x y z} ; x, y, z \leftarrow Z_{q}^{*}, Q, x Q, y Q, z Q \leftarrow G_{1}\right] \leq \varepsilon .
$$

\section{Proposed TPAE Construction}

Using the operation and properties of bilinear pairing, this section is going to present the designed TPAE construction. At first, the roles of participated entities will be briefly described. Then we display the definition of constituted algorithms followed by a secure construction.

\subsection{Participated Entity}

A general TPAE construction has three participated entities, which cover an original signer and two designated receivers. The former will produce an authenticated ciphertext. Then, both specified receivers can independently decrypt the obtained ciphertext and inspect the validity of embedded signature. A TPAE scheme is said to be correct provided that a signer creates a valid authenticated ciphertext and merely the specified receivers have the privilege to decrypt it and check the validity of embedded signature. Each of the designated receivers could exhibit a transformed signature suitable for public proof when encountering a repudiation dispute, too.

\subsection{Constituted Algorithms}

The designed TPAE scheme can be divided into four algorithms. We describe the definition of each algorithm as follows.

Setup: This algorithm is used to initialize the constructed system by taking a security parameter $k$ and then generates public parameters params.

Reg_U: The input of this algorithm is an index $i$ while the corresponding output includes a private-public keypair $\left(x_{i}, Y_{i}\right)$ together with a public key certificate Cert $t_{i}$.

ESign: The input of this algorithm includes $m, x_{s}, Y_{a}$, and $Y_{b}$, which separately represents a message, a signing key, and two verification keys. It will finally generate a corresponding authenticated ciphertext $\delta$.

EVerify: The input of this algorithm includes $\delta, x_{a}, I D_{s}, I D_{a}$, and $I D_{b}$, which separately denote a ciphertext, a decryption key, and the identities of one signer and two recipients. The output could be either a transformed signature $\Omega$ with the original message $m$ or an error symbol $\perp$. The latter case occurs if the input contains a false ciphertext.

\subsection{Substantial Construction}

Setup: Let $k$ be a security parameter of the input. The algorithm first selects two groups, i.e., $G_{1}$ and $G_{2}$, which have an identical order of prime $q$. There is a generator $P$ in the group $G_{1}$ and a bilinear map $e$ is defined as $G_{1} \times G_{1} \rightarrow G_{2}$. Assume that $h_{1}:\{0,1\}^{k} \times G_{1}{ }^{2} \rightarrow Z_{q}, h_{2}: G_{1}{ }^{3} \rightarrow\{0,1\}^{k}$ and $h_{3}: G_{2} \rightarrow$ $G_{1}$ are collision resistant hash functions. The algorithm outputs public params which are composed of $G_{1}, G_{2}, q, P$, and $e$ along with three hash functions.

Reg_U: Given an index $i$, the algorithm randomly chooses an integer $x_{i} \in Z_{q}$ as the corresponding private key and then calculates the value $Y_{i}=x_{i} P$ to be its public key. Note that a public key certificate named Cert ${ }_{i}$ is also returned by employing the standard of X.509 [32]. 
ESign: To produce an authenticated ciphertext for a message $m$ in relation to two verification keys $\left(Y_{a}\right.$ and $\left.Y_{b}\right)$ and a signing key $x_{s}$, the algorithm chooses $w \in Z_{q}^{*}$ to compute

$$
\begin{gathered}
W=w P, \\
T=h_{3}\left(e\left(w Y_{a}, Y_{b}\right)\right), \\
\sigma=\left(x_{s}+h_{1}(m, W, T)\right)^{-1} W, \\
c=m \oplus h_{2}(W, \sigma, T),
\end{gathered}
$$

and then outputs the authenticated ciphertext $\delta=(W, \sigma, c)$.

Note that in Equation (4), the ciphertext parameter $c$ is computed by XOR-ing the message $m$ and the hash result of $h_{2}(W, \sigma, T)$. This implies that the original message has to be split into $k$-bit blocks for facilitating the XOR operation. Therefore, the ciphertext parameter $c$ is constituted by concatenating all XORed blocks.

EVerify: Given an authenticated ciphertext $\delta=(W, \sigma, c)$, one private key $x_{i}$ (for $i \in\{a, b\}$ ) of participated receivers and the signing key $Y_{s}$, this algorithm can easily derive

$$
T=h_{3}\left(e\left(x_{a} W, Y_{b}\right)\right)=h_{3}\left(e\left(Y_{a}, x_{b} W\right)\right),
$$

to decrypt the original message $m$ as

$$
m=c \oplus h_{2}(W, \sigma, T)
$$

and then inspects the embedded redundancy of $m$. The algorithm further checks its signature by testing whether

$$
e\left(\sigma, Y_{s}+h_{1}(m, W, T) P\right)=e(W, P) .
$$

When the above equality is fulfilled, the algorithm returns the decrypted message $m$ together with a transformed signature $\Omega=(W, \sigma, T)$. If not, an error symbol $\perp$ is outputted to denote the false ciphertext.

The correctness of Equations (6) and (7) could be confirmed by the following derivations. In Equation (6), the right side can be written as

$$
\begin{aligned}
& c \oplus h_{2}(W, \sigma, T) \\
= & c \oplus h_{2}\left(W, \sigma, h_{3}\left(e\left(x_{a} W, Y_{b}\right)\right)\right) \\
= & c \oplus h_{2}\left(W, \sigma, h_{3}\left(e\left(x_{a} t P, Y_{b}\right)\right)\right) \\
= & c \oplus h_{2}\left(W, \sigma, h_{3}\left(e\left(w Y_{a}, Y_{b}\right)\right)\right) \\
= & c \oplus h_{2}(W, \sigma, T) \\
= & m
\end{aligned}
$$

(by Equation (5))

(by Equation (1))

(by Equation (2))

(by Equation (4))

which is just the left side of Equation (6).

In Equation (7), the left side can be written as

$$
\begin{aligned}
& e\left(\sigma, Y_{s}+h_{1}(m, W, T) P\right) \\
= & e\left(\left(x_{s}+h_{1}(m, W, T)\right)^{-1} W, Y_{s}+h_{1}(m, W, T) P\right) \\
= & e\left(\left(x_{s}+h_{1}(m, W, T)\right)^{-1} W,\left(x_{s}+h_{1}(m, W, T)\right) P\right) \\
= & e(W, P)
\end{aligned}
$$

which is the right side of Equation (7).

\section{Security Model and Proof}

To prove the security of our TPAE scheme, we first revisit the general security models of confidentiality and unforgeability below. 
Definition 1 (Requirement of IND-CCA2). The proposed TPAE scheme satisfies the characteristic of indistinguishability for the confidentiality requirement provided that no probabilistic polynomial-time (PPT) adversary $\mathcal{A}$ plotting adaptive chosen ciphertext attacks has a non-negligible advantage to beat a player $\mathcal{B}$ acting as a challenger in the following game.

Setup $\left(1^{k}\right)$ : By initializing the $\operatorname{Setup}\left(1^{k}\right)$ algorithm, the challenger $\mathcal{B}$ first provides the adversary $\mathcal{A}$ with public params.

Phase 1: The querying capability of adversary $\mathcal{A}$ consists of the following oracles.

Reg_U query: $\mathcal{A}$ could choose an index $i$ to request its Reg_U query. $\mathcal{B}$ would run the Reg_U algorithm and return the output of $\left(Y_{i}\right.$, Cert $\left._{i}\right)$.

ESign query: $\mathcal{A}$ could request an ESign query for his/her chosen $m, Y_{s}, Y_{a}$, and $Y_{b}$. B outputs a corresponding authenticated ciphertext $\delta$ to $\mathcal{A}$.

EVerify query: $\mathcal{A}$ could request an EVerify query for his/her chosen ciphertext $\delta$. $\mathcal{B}$ would send either an error symbol $\perp$ or a message $m$ along with its transformed signature $\Omega$ to $\mathcal{A}$.

Challenge: After requesting several queries, the adversary $\mathcal{A}$ would create messages, say, $m_{0}$ and $m_{1}$, where $\left|m_{0}\right|=\left|m_{1}\right|$. Then $\mathcal{B}$ determines $\lambda \leftarrow\{0,1\}$ by flipping an internal coin. An authenticated ciphertext $\delta^{*}$ on $m_{\lambda}$ is also computed as a challenge designated for $\mathcal{A}$.

Phase 2: In this phase, the adversary $\mathcal{A}$ continues to request new queries, but does not include any EVerify query on the target challenge.

Guess: At last, we say that the adversary $\mathcal{A}$ is the winner of this game provided that his/her guessed bit $\lambda^{\prime}=\lambda$. The advantage of $\mathcal{A}$, denoted as $A d v(\mathcal{A})$, could be written as $\left|\operatorname{Pr}\left[\lambda^{\prime}=\lambda\right]-0.5\right|$.

Definition 2 (Requirement of EF-CMA). The proposed TPAE scheme satisfies the characteristic of existential unforgeability provided that no PPT adversary $\mathcal{A}$ plotting adaptive chosen-message attacks has a non-negligible advantage to beat a player $\mathcal{B}$ acting as a challenger in the following game:

Setup $\left(1^{k}\right)$ : By initializing the $\operatorname{Setup}\left(1^{k}\right)$ algorithm, the challenger $\mathcal{B}$ first provides the adversary $\mathcal{A}$ with public params.

Phase 1: The querying capability of adversary $\mathcal{A}$ consists of Reg_U and ESign oracles just like those described in Definition 1.

Forgery: At the end of this game, we say that the winner is $\mathcal{A}$ if he/she outputs a valid $\delta^{*}$ on an arbitrarily chosen $m^{*}$. It should be noted that $\delta^{*}$ could not be obtained via an ESign oracle.

We then adopt the techniques of random oracle proof models to formally show that the proposed mechanism satisfies the security of Definitions 1 and 2.

Theorem 1 (Proof of IND-CCA2). It is said that the proposed TPAE scheme is $\left(t, q_{h 1}, q_{h 2}, q_{h 3}, q_{\text {Reg_ }} U, q_{E S i g n}\right.$, $\left.q_{E V e r i f y}, \varepsilon\right)$-secure in the requirement of IND-CCA2 when no PPT adversary has the non-negligible advantage $\varepsilon^{\prime}$ to solve the BDHP within the running time $t^{\prime}$, where

$$
\begin{gathered}
\varepsilon^{\prime} \geq\left(\frac{1}{q_{h_{3}}}\right)\left(2 \varepsilon-\frac{q_{\text {EVerify }}}{2^{k}}\right), \\
t^{\prime} \approx t+t_{\lambda}\left(2 q_{\text {EVerify }}\right) .
\end{gathered}
$$

Here, $t_{\lambda}$ represents the required computation time of a bilinear map.

Proof. We first assume that within the running time, $t$, there is a PPT adversary, $\mathcal{A}$, whose capability is sufficient to break the proposed TPAE construction by plotting adaptive chosen ciphertext attacks. The advantage of the adversary $\mathcal{A}$ is $\varepsilon$ and the maximum query times of oracle $i$ is denoted as $q_{i}$. By utilizing $\mathcal{A}$ as a subroutine, it enables us to create a new algorithm, say $\mathcal{B}$, to break the assumption of $\mathrm{BDH}$ within the expected time $t^{\prime}$ and the success probability is $\varepsilon^{\prime}$. Let $P, x P, y P$, and $z P$ be the 
inputted BDHP instance for $\mathcal{B}$ and the desired output would be $e(P, P)^{x y z}$. In the following interactive processes, $\mathcal{B}$ acts as a challenger to answer queries submitted by $\mathcal{A}$.

Setup: By performing the initial $\operatorname{Setup}\left(1^{k}\right)$ algorithm, the challenger $\mathcal{B}$ provides the adversary $\mathcal{A}$ with public params $=\left\{G_{1}, G_{2}, q, P, e\right\}$.

Phase 1: In the beginning, $\mathcal{A}$ designates $\left(I D_{s}, I D_{a}\right.$, and $\left.I D_{b}\right)$ as the identities of the signer and two designated verifiers, and could adaptively request queries stated below.

$h_{1}$ oracle: $\mathcal{A}$ could submit an $h_{1}(m, W, T)$ oracle to get the value $v_{1} \in_{R} Z_{q}$. A record of $\left(m, W, T, v_{1}\right)$ would also be written into a maintained $h_{1}$-list by $\mathcal{B}$.

$h_{2}$ oracle: $\mathcal{A}$ could submit an $h_{2}(W, \sigma, T)$ oracle to get the value $v_{2} \in_{R}\{0,1\}^{k}$. A record of $(W, \sigma$, $\left.T, v_{2}\right)$ would also be written into a maintained $h_{2}$-list by $\mathcal{B}$.

$h_{3}$ oracle: $\mathcal{A}$ could submit an $h_{3}(E)$ oracle to get the value $v_{3} \in_{R} G_{1}$. A record of $\left(E, v_{3}\right)$ would also be written into a maintained $h_{3}$-list by $\mathcal{B}$.

Reg_U query: $\mathcal{A}$ could choose an index $i$ to request its Reg_U query. If $i=a, \mathcal{B}$ sends $\left(Y_{a}=x P\right.$, $\left.\operatorname{Cert}_{a}\right)$ to $\mathcal{A}$. If $i=b, \mathcal{B}$ returs $\left(Y_{b}=y P\right.$, Cert $\left._{b}\right)$ to $\mathcal{A}$. When $i=s, \mathcal{B}$ calls the Reg_U algorithm to get $\left(x_{s}, Y_{s}\right.$, Cert $\left._{s}\right)$ and then returns $\left(Y_{s}, \operatorname{Cert}_{s}\right)$ to $\mathcal{A}$.

ESign query: $\mathcal{A}$ could request an ESign query on an arbitrarily chosen message $m$. $\mathcal{B}$ would run the ESign algorithm to return the corresponding result.

EVerify query: $\mathcal{A}$ could request an EVerify query on a ciphertext $\delta=(W, \sigma, c) . \mathcal{B}$ utilizes $(W, \sigma)$ as keywords to search the $h_{2}$-list for all matched $v_{2^{\prime}}$ s. If one of matched $v_{2^{\prime}} \mathrm{s}$ satisfies that $e\left(\sigma, Y_{s}+h_{1}(c \oplus\right.$ $\left.\left.v_{2}, W, T\right) P\right)=e(W, P), \mathcal{B}$ returns $\left(c \oplus v_{2}, W, \sigma, T\right)$. If not, $\mathcal{A}$ will receive an error symbol.

Challenge: After requesting several queries, the adversary $\mathcal{A}$ would create messages, say $m_{0}$ and $m_{1}$, where $\left|m_{0}\right|=\left|m_{1}\right|$. Then $\mathcal{B}$ determines $\lambda \leftarrow\{0,1\}$ by flipping an internal coin and computes a ciphertext $\delta^{*}$ for the selected $m_{\lambda}$ with the following steps:

Step 1 Randomly choose $v_{1} \in Z_{q}$ along with $v_{2} \in\{0,1\}^{k}$;

Step 2 Let $W^{*}=z P$;

Step 3 Compute $\sigma^{*}=\left(x_{S}+v_{1}\right)^{-1} W^{*}$ and $c^{*}=m_{\lambda} \oplus v_{2}$;

Step 4 Add the record of $\left(m_{\lambda}, W^{*}\right.$, null, $\left.v_{1}\right)$ into $h_{1}$-list;

Step 5 Add the record of $\left(W^{*}, \sigma^{*}\right.$, null, $\left.v_{2}\right)$ into $h_{2}$-list. The ciphertext $\delta^{*}=\left(W^{*}, \sigma^{*}, c^{*}\right)$ is served as a target challenge for $\mathcal{A}$.

Phase 2: In this phase, the adversary $\mathcal{A}$ continues to request new queries, but does not include any EVerify queries on the target challenge $\delta^{*}$.

Analysis of the game: In this game interaction, it is possible for the adversary $\mathcal{A}$ to get an error symbol with respect to an EVerify query on some valid $\delta=(W, \sigma, c)$. Such an event will happen in the case that the corresponding $h_{2}(W, \sigma, T)$ oracle had never been made before. We express this event as EVerify_Fat and $\operatorname{Pr}\left[\right.$ EVerify_Fat] during the entire simulation game is not greater than $\frac{q_{E V \text { erify }}}{2^{k}}$, as $\mathcal{A}$ can issue at most $q_{E V \text { erify }}$ EVerify queries. Besides, in the challenge phase, $\mathcal{B}$ sets $W^{*}=z P$, which infers that the component $Z^{*}$ is formulated as $h_{3}(e(x(z P), y P))=h_{3}\left(e(P, P)^{x y z}\right)$. If the adversary $\mathcal{A}$ queries an $h_{3}$ oracle on the value $e(P, P)^{x y z}$ during the second phase, the simulation game would accidentically terminate. We denote such an event as $\mathrm{QH}_{3}{ }^{*}$ and let PSG be the event of perfect simulation game. When the event PSG occurs, $\mathcal{A}$ has no better change to guess $\lambda$, i.e.,

$$
\operatorname{Pr}\left[\lambda^{\prime}=\lambda \mid \mathrm{PSG}\right]=0.5 \text {. }
$$

Modified from the probability event of $\operatorname{Pr}\left[\lambda^{\prime}=\lambda\right]$, it could be obtained that

$$
\begin{gathered}
\operatorname{Pr}\left[\lambda^{\prime}=\lambda\right]=\operatorname{Pr}\left[\lambda^{\prime}=\lambda \mid \text { PSG }\right] \operatorname{Pr}[\mathrm{PSG}]+\operatorname{Pr}\left[\lambda^{\prime}=\lambda \mid \neg \mathrm{PSG}\right] \operatorname{Pr}[\neg \mathrm{PSG}] \\
\leq 0.5 \operatorname{Pr}[\mathrm{PSG}]+\operatorname{Pr}[\neg \mathrm{PSG}]
\end{gathered}
$$

(by Equation 8) 


$$
\begin{gathered}
=0.5(1-\operatorname{Pr}[\neg \text { PSG }])+\operatorname{Pr}[\neg \text { PSG }] \\
=0.5+0.5 \operatorname{Pr}[\neg \text { PSG }] .
\end{gathered}
$$

Additionally, it could also be learned that

$$
\begin{aligned}
\operatorname{Pr}\left[\lambda^{\prime}=\lambda\right. & \geq \operatorname{Pr}\left[\lambda^{\prime}=\lambda \mid \mathrm{PSG}\right] \operatorname{Pr}[\mathrm{PSG}] \\
& =0.5(1-\operatorname{Pr}[\neg \mathrm{PSG}]) \\
& =0.5-0.5 \operatorname{Pr}[\neg \mathrm{PSG}] .
\end{aligned}
$$

Integrating inequalities (9) with (10), we have

$$
\left|\operatorname{Pr}\left[\lambda^{\prime}=\lambda\right]-0.5\right| \leq 0.5 \operatorname{Pr}[\neg \mathrm{PSG}] .
$$

According to the proof assumption, the adversary $\mathcal{A}$ is sufficient to break our TPAE construction with a non-negligible chance $\varepsilon$, which indicates that

$$
\begin{aligned}
\varepsilon & =\left|\operatorname{Pr}\left[\lambda^{\prime}=\lambda\right]-0.5\right| \\
& \leq 0.5 \operatorname{Pr}[\neg \operatorname{PSG}] \\
& =0.5\left(\operatorname{Pr}\left[\mathrm{QH}_{3}{ }^{*} \vee \text { EVerify_Fat }\right]\right) \\
& \leq 0.5\left(\operatorname{Pr}\left[\mathrm{QH}_{3}{ }^{*}\right]+\operatorname{Pr}[\text { EVerify_Fat }]\right) .
\end{aligned}
$$

Further rewrite this inequality and it will have that

$$
\begin{aligned}
& \operatorname{Pr}\left[\mathrm{QH}_{3}{ }^{*}\right] \geq 2 \varepsilon-\operatorname{Pr}[\text { EVerify_Fat }] \\
& \geq 2 \varepsilon-\frac{q_{\text {EVerify }}}{2^{k}} .
\end{aligned}
$$

When the event $\mathrm{QH}_{3}{ }^{*}$ happens, we claim that $e(P, P)^{x y z}$ would be contained in a record of $h_{3}$-list. For that reason, we could mean that the advantage of the algorithm $\mathcal{B}$ for breaking the designated BDHP instance is $\varepsilon^{\prime} \geq\left(\frac{1}{q_{h_{3}}}\right)\left(2 \varepsilon-\frac{q_{E V} \text { erify }}{2^{k}}\right)$. The expected running time of $\mathcal{B}$ is calculated as $t^{\prime} \approx t+$ $t_{\lambda}\left(2 q_{E V e r i f y}\right)$.

Theorem 2 (Proof of EF-CMA). It is said that the proposed TPAE scheme is $\left(t, q_{h 1}, q_{h 2}, q_{h 3}, q_{\text {Reg_ }}\right.$, $q_{E S i g n}$, $\varepsilon$ )-secure in the requirement of EF-CMA when no PPT adversary having the non-negligible advantage $\varepsilon^{\prime}$ solves the ECDLP within the running time $t^{\prime}$, where

$$
\begin{gathered}
\varepsilon^{\prime} \geq 10\left(q_{E S i g n}+1\right)\left(q_{E S i g n}+q_{h 1}\right) / 2^{k} \\
t^{\prime} \leq 120686 q_{h 1} t / \varepsilon
\end{gathered}
$$

Proof. We first assume that within the running time $t$, there is a PPT adversary, $\mathcal{A}$, whose capability is sufficient to break the proposed TPAE construction by plotting adaptive chosen message attacks. The advantage of the adversary $\mathcal{A}$ is $\varepsilon$ and the maximum query times of oracle $i$ is denoted as $q_{i}$. By utilizing $\mathcal{A}$ as a subroutine, it enables us to create a new algorithm, say $\mathcal{B}$, to break the assumption of ECDL within the expected time $t^{\prime}$ and the success probability is $\varepsilon^{\prime}$. Let $(P, z P)$ be the inputted ECDLP instance for $\mathcal{B}$ and the purpose is to obtain $z$. In this proof, we use the technique of Forking Lemma [33] and $\mathcal{B}$ acts as a challenger to answer queries submitted by $\mathcal{A}$.

Setup: By performing the initial Setup $\left(1^{k}\right)$ algorithm, the challenger $\mathcal{B}$ provides the adversary $\mathcal{A}$ with public params $=\left\{G_{1}, G_{2}, q, P, e\right\}$ and a random tape which is constituted of a series of random 
bits. Given public params and an identical random tape, $\mathcal{B}$ would play two rounds of games with the adversary $\mathcal{A}$ below.

Phase 1: In the beginning, $\mathcal{A}$ designates $\left(I D_{s}, I D_{a}\right.$, and $\left.I D_{b}\right)$ as the selected identities of the signer together with two intended verifiers, and then adaptively makes new queries as follows. For all $h_{i}$ $(i \in\{1,2,3\})$ oracles, $\mathcal{B}$ returns as those defined in Theorem 1.

Reg_U query: When $\mathcal{A}$ requests an Reg_U query on the index $i=s, \mathcal{B}$ directly sends $\left(Y_{s}=z P, \operatorname{Cert}_{s}\right)$ to $\mathcal{A}$. Otherwise, $\mathcal{B}$ calls the Reg_U algorithm for getting $\left(x_{i}, Y_{i}\right.$, and Cert $\left.{ }_{i}\right)$ and then returns $\left(Y_{i}\right.$, Cert $\left._{i}\right)$ to $\mathcal{A}$.

ESign query: When $\mathcal{A}$ submits an ESign query on his/her chosen $m, Y_{s}, Y_{a}$, and $Y_{b}, \mathcal{B}$ performs the subsequent procedures:

Step 1 Randomly pick two integers $d, v_{1} \in Z_{q}^{*}$;

Step 2 Run the Reg_U algorithm to get $\left(x_{a}, Y_{a}, \operatorname{Cert}_{a}\right)$ and $\left(x_{b}, Y_{b}\right.$, Cert $\left.b\right)$;

Step 3 Compute

$$
\begin{aligned}
& \sigma=d P \\
& W=d(z P)+v_{1} d P, \\
& T=h_{3}\left(e\left(x_{a} W, Y_{b}\right)\right) \\
& c=m \oplus h_{2}(W, \sigma, T)
\end{aligned}
$$

Step 4 Add the entry $\left(m, W, T, v_{1}\right)$ into $h_{1}$-list; The ciphertext $\delta=(W, \sigma, c)$ is then returned to $\mathcal{A}$.

Analysis of the game: In the above simulation, it is obvious that the ESign query always returns a valid authenticated ciphertext, such that the adversary $\mathcal{A}$ is incapable of distinguishing it from the one outputted by the real scheme. When $\mathcal{A}$ successfully forges a fresh ciphertext $\delta=(W, \sigma, c)$ for the message $m$ denoted by the event $\mathrm{PF}$, we obtain $\operatorname{Pr}[\mathrm{PF}]=\varepsilon$ as $\mathcal{A}$ owns a non-negligible chance $\varepsilon$ of breaking our TPAE construction. Now we evaluate the probability that $\mathcal{A}$ does not query the corresponding $h_{1}$ oracle and we know that $\operatorname{Pr}\left[\neg \mathrm{QH}_{1}\right] \leq 2^{-k}$. Hence, it could be further expressed that the probability of $\mathcal{A}$ to generate a valid ciphertext $\delta=(W, \sigma, c)$ following making the corresponding $h_{1}(m, W, T)$ oracle to be $\operatorname{Pr}\left[\mathrm{PF} \wedge \mathrm{QH}_{1}\right] \geq\left(\varepsilon-1 / 2^{k}\right)$. Afterwards, $\mathcal{B}$ plays the second round with $\mathcal{A}$ in the same environment. Because the inputted tape is formed by identical series of randomized bits, the challenger $\mathcal{B}$ is able to anticipate $\mathcal{A}^{\prime}$ s next query. According to the responses made in first run, $\mathcal{B}$ returns the same results until $\mathcal{A}$ queries the critical $h_{1}(m, W, T)$ oracle. Instead of returning original $v_{1}, \mathcal{B}$ outputs a new value $v_{1}{ }^{*}$. By the Forking Lemma, if $\mathcal{A}$ finally forges a valid ciphertext $\delta^{*}=\left(W, \sigma^{*}\right.$, $\left.c^{*}\right)$ with $h_{1}(m, W, T)=v_{1}^{*}, \mathcal{B}$ can solve the ECDLP. Concretely speaking, when the adversary $\mathcal{A}$ finally outputs two valid ciphertext $\left(\delta, \delta^{*}\right)$ with $h_{1}(m, W, T) \neq h_{1}^{*}(m, W, T)$, we can obtain two equations:

$$
\begin{gathered}
\sigma=\left(x_{s}+h_{1}(m, W, T)\right)^{-1} W=\left(z+v_{1}\right)^{-1} W, \\
\sigma^{*}=\left(x_{s}+h_{1}^{*}(m, W, T)\right)^{-1} W=\left(z+v_{1}^{*}\right)^{-1} W .
\end{gathered}
$$

Thus, the ECDLP instance can be solved by computing $z=\frac{\sigma^{*} v_{1}{ }^{*}-\sigma v_{1}}{\sigma-\sigma^{*}}$. We could evaluate $\mathcal{B}^{\prime}$ s success probability after two simulation games as $\varepsilon \geq 10\left(q_{E S i g n}+1\right)\left(q_{E S i g n}+q_{h 1}\right) / 2^{k}$ and the expected running time spent by $\mathcal{B}$ is $t^{\prime} \leq 120686 q_{h 1} t / \varepsilon$.

Note that an adversary might try to derive a valid ciphertext if he/she has access to multiple ciphertexts. Yet, each valid ciphertext will also contain a valid signature which is randomly generated and protected by a secret value chosen by the signer. Consequently, it is computationally infeasible for any adversary to plot the forgery attacks from multiple known ciphertexts.

\section{Performance Evaluation}

In a three-party communication environment, a party usually has to transmit the same message to the other two parties. When the message is encrypted with traditional asymmetric approaches, the sender is required to run the encryption process twice for two recipients who do not want to share 
their private keys with each other. In such a way, a sender engaged in three-party communication environments often incurs higher computational costs. From the above computational perspective, we will show that the proposed scheme is better than previous single-user setting mechanisms.

As our protocol is implemented on pairing-based systems, we only consider some time-consuming operations such as bilinear map, exponentiation, and scalar multiplication. The operations of XOR, addition and one-way hash function are ignored. We present the used symbols as Table 1 . Table 2 is the summary of computational comparisons among our TPAE scheme and thee similar protocols involving Lee et al.'s (Lee10) [34], the Hsu-Lin (Hsu14) [24], and the Islam-Biswas (Islam13) [35]. In order to obtain more united comparison results, we adopt Cao et al.'s experimental figures [36] to transform the above computation into approximate running time as Figures 1-3. Specifically, the computation of a bilinear pairing, exponentiation and scalar multiplication will take approximately $20.01 \mathrm{~ms}, 11.2 \mathrm{~ms}$, and $6.38 \mathrm{~ms}$, respectively.

Table 1. The used symbols.

\begin{tabular}{cc}
\hline Symbol & Description \\
\hline$C_{1}$ & To execute a bilinear pairing computation \\
$C_{2}$ & To execute an exponentiation computation over $G_{2}$ \\
$C_{3}$ & To execute a scalar multiplication over $G_{1}$ \\
\hline
\end{tabular}

Table 2. Computational comparison in three-party communication environments.

\begin{tabular}{cccc}
\hline & Sender & Each Recipient & Entire Scheme \\
\hline Lee10 & $4 C_{1}+4 C_{3}$ & $2 C_{1}+C_{3}$ & $8 C_{1}+6 C_{3}$ \\
Hsu14 & $2 C_{1}+8 C_{3}$ & $3 C_{1}+3 C_{3}$ & $8 C_{1}+14 C_{3}$ \\
Islam13 & $6 C_{1}+6 C_{3}+2 C_{2}$ & $C_{1}+C_{3}+C_{2}$ & $8 C_{1}+8 C_{3}+4 C_{2}$ \\
This paper & $C_{1}+3 T_{M}$ & $3 C_{1}+2 C_{3}$ & $7 C_{1}+7 C_{3}$ \\
\hline
\end{tabular}

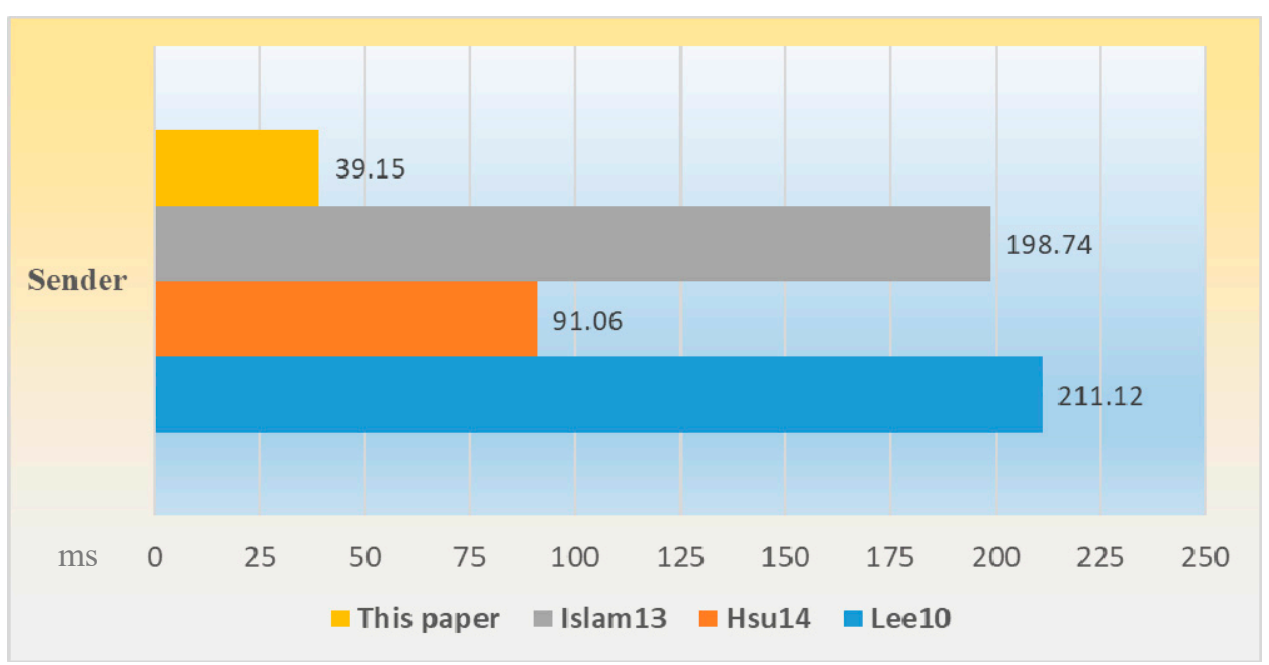

Figure 1. Comparison of approximate running time for sender in three-party communication environments.

In Figure 1, a sender of the proposed scheme has to spend about $39.15 \mathrm{~ms}$ for generating a ciphertext intended for two recipients. The required time is minimal among all compared mechanisms. Although in Figure 2, each recipient's running time of both Lee et al.'s [34] and the Islam-Biswas [35] is better than that of ours and the proposed scheme still exhibits better performance as a whole in Figure 3. Accordingly, it is evident that our proposed scheme outperforms compared ones from either the sender side or the entire mechanism. 


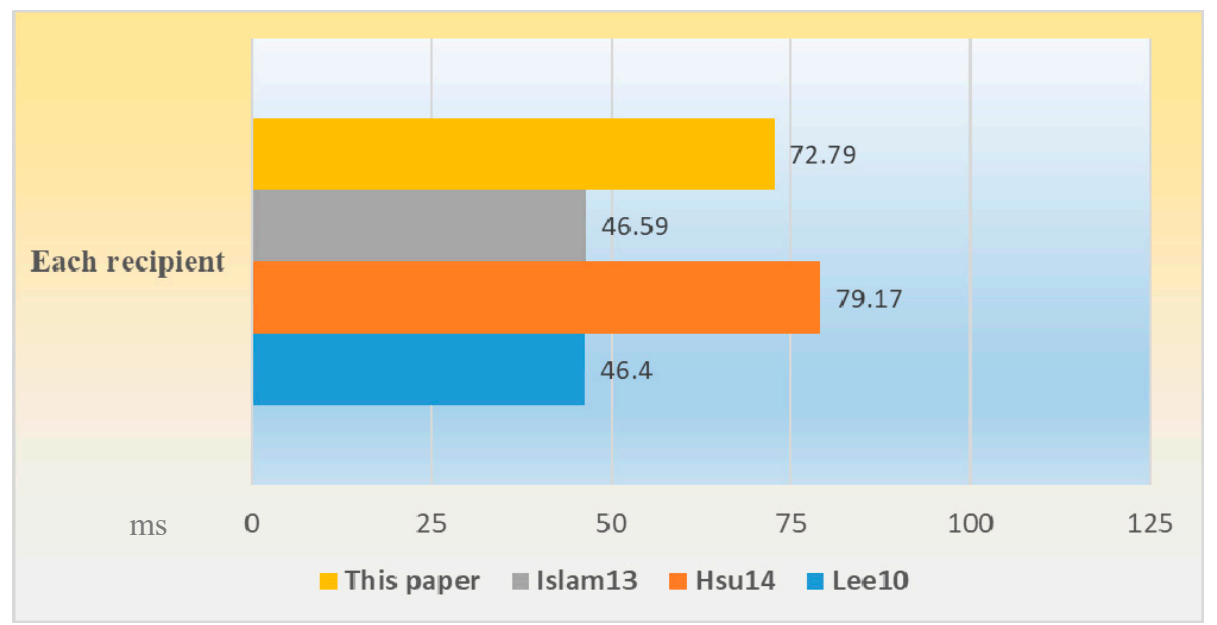

Figure 2. Comparison of approximate running time for each recipient in three-party communication environments.

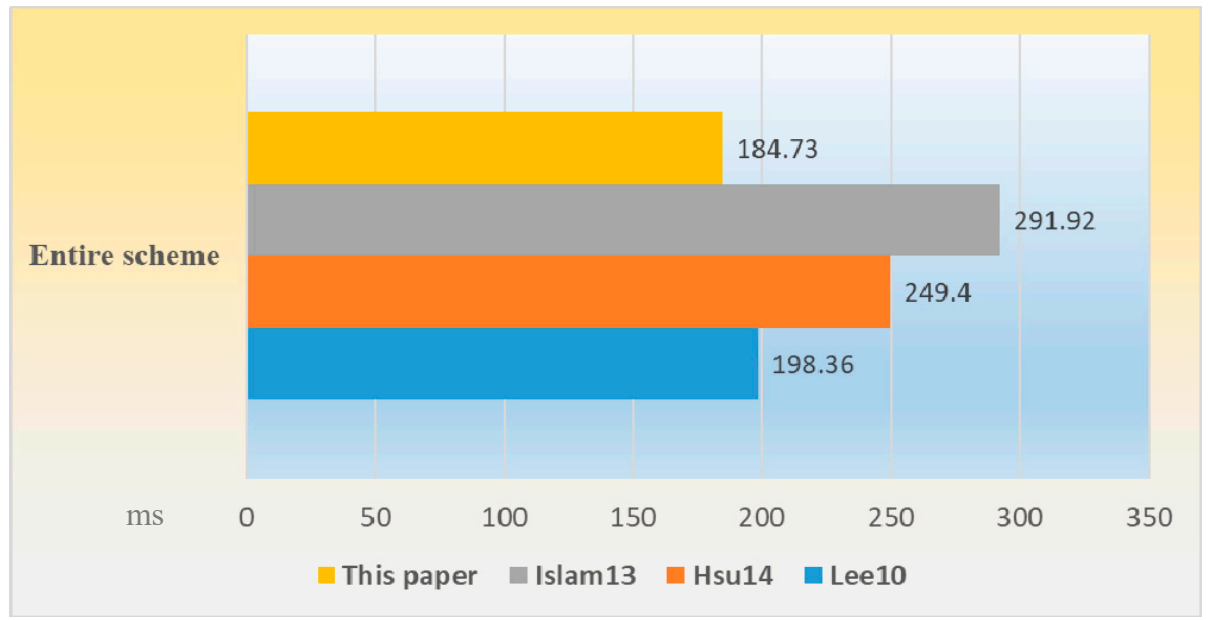

Figure 3. Comparison of approximate running time for entire scheme in three-party communication environments.

\section{Conclusions}

AE schemes with convertibility have found numerous applications in online transactions such as e-auction and confidential contract signing. In this paper, we focused on the applications of three-party communication environments and proposed a novel three-party AE (TPAE) scheme. Unlike most group-oriented AE mechanisms which require cooperative verification, our TPAE scheme allows two designated recipients to solely decrypt acquired ciphertext along with inspecting the recovered signature without utilizing shared secrets. Moreover, the signature conversion process also exhibits the property of computation-free and can be carried out by each designated recipient alone. To make certain the realistic feasibility, the requirement of IND-CCA2 and that of EF-CMA for our construction are both fulfilled in the formal proof model of random oracles. Compared to previous similar protocols in terms of computational efforts, the designed approach is particularly appealing to the three-party applications.

Author Contributions: H.-Y.L. wrote the original draft.

Funding: This work was supported in part by the Ministry of Science and Technology of Republic of China under the contract number MOST 107-2221-E-019-017.

Conflicts of Interest: The author declares no conflicts of interest.

Ethical Approval: This article does not contain any studies with human participants or animals performed by the author. 


\section{References}

1. Diffie, W.; Hellman, M. New Directions in Cryptography. IEEE Trans. Inf. Theory 1976, IT-22, $644-654$. [CrossRef]

2. ElGamal, T. A Public Key Cryptosystem and a Signature Scheme Based on Discrete Logarithms. IEEE Trans. Inf. Theory 1985, IT-31, 469-472. [CrossRef]

3. Rivest, R.; Shamir, A.; Adleman, L. A Method for Obtaining Digital Signatures and Public-Key Cryptosystems. Commun. ACM 1978, 21, 120-126. [CrossRef]

4. Sekhar, M.R. Signatures Scheme with Message Recovery and Its Applications. Int. J. Comput. Math. 2004, 81, 285-289. [CrossRef]

5. Schneider, S. Formal Analysis of a Non-Repudiation Protocol. In Proceedings of the 11th IEEE Computer Security Foundations Workshop, Rockport, MA, USA, 9-11 June 1998; IEEE Press: Piscataway, NJ, USA, 1998; pp. 54-65.

6. Hou, F.; Wang, Z.; Tang, Y.; Liu, Z. Protecting Integrity and Confidentiality for Data Communication. In Proceedings of the 9th International Symposium on Computers and Communications (ISCC'04), Alexandria, Egypt, 28 June-1 July 2004; pp. 357-362.

7. Jacob, J. A Uniform Presentation of Confidentiality Properties. IEEE Trans. Softw. Eng. 1991, 17, 1186-1194. [CrossRef]

8. Horster, P.; Michel, M.; Peterson, H. Authenticated Encryption Schemes with Low Communication Costs. Electron. Lett. 1994, 30, 1212-1213. [CrossRef]

9. Stallings, W. Cryptography and Network Security: Principles and Practices, 7th ed.; Pearson: London, UK, 2017.

10. Araki, S.; Uehara, S.; Imamura, K. The Limited Verifier Signature and Its Application. IEICE Trans. Fundam. Electron. Comput. Sci. 1999, E82-A, 63-68.

11. Zhang, F.; Kim, K. A Universal Forgery on Araki et al.'s Convertible Limited Verifier Signature Scheme. IEICE Trans. Fundam. Electron. Comput. Sci. 2003, E86-A, 515-516.

12. Wu, T.S.; Hsu, C.L. Convertible Authenticated Encryption Scheme. J. Syst. Softw. 2002, 62, 205-209. [CrossRef]

13. Huang, H.F.; Chang, C.C. An Efficient Convertible Authenticated Encryption Scheme and Its Variant. In Proceedings of the 5th International Conference on Information and Communications Security (ICICS 2003), Huhehaote, China, 10-13 October 2003; pp. 382-392.

14. Lv, J.; Wang, X.; Kim, K. Practical Convertible Authenticated Encryption Schemes Using Self-Certified Public Keys. Appl. Math. Comput. 2005, 169, 1285-1297. [CrossRef]

15. Yang, F.Y. A Secure Scheme for Authenticated Encryption. Cryptology ePrint Archive, Report 2005/456. 2005. Available online: http://eprint.iacr.org/2005/456 (accessed on 11 February 2019).

16. Chien, H.Y. Selectively Convertible Authenticated Encryption in the Random Oracle Model. Comput. J. 2008, 51, 419-434. [CrossRef]

17. Lee, C.C.; Hwang, M.S.; Tzeng, S.F. A New Convertible Authenticated Encryption Scheme Based on the ElGamal Cryptosystem. Int. J. Found. Comput. Sci. 2009, 20, 351-359. [CrossRef]

18. Wu, T.S.; Lin, H.Y. Secure Convertible Authenticated Encryption Scheme Based on RSA. Informatica-Lithuan 2009, 33, 481-486.

19. Lin, H.Y.; Hsu, C.L. A Novel Identity-Based Key-Insulated Convertible Authenticated Encryption Scheme. Int. J. Found. Comput. Sci. 2011, 22, 739-756. [CrossRef]

20. Hsu, C.L.; Lin, H.Y. New Identity-Based Key-Insulated Convertible Multi-Authenticated Encryption Scheme. J. Netw. Comput. Appl. 2011, 34, 1724-1731. [CrossRef]

21. Lin, H.Y. Group-Oriented Data Access Structure Using Threshold-CAE Scheme and Its Extension. Inf. Technol. Control 2014, 43, 252-263. [CrossRef]

22. Lin, H.Y. "PCMAE: A Proxy Convertible Multi-AE Scheme and Its Variant. Inf. Technol. Control 2017, 46, 530-545. [CrossRef]

23. Wu, T.S.; Lin, H.Y.; Ting, P.Y. A Publicly Verifiable PCAE Scheme for Confidential Applications with Proxy Delegation. Trans. Emerg. Telecommun. Technol. 2012, 23, 172-185. [CrossRef]

24. Hsu, C.L.; Lin, H.Y. Convertible Authenticated Encryption Scheme with Hierarchical Access Control. Appl. Math. Inf. Sci. 2014, 8, 1239-1246. [CrossRef]

25. Lin, H.Y.; Hsu, C.L.; Huang, S.K. Improved Convertible Authenticated Encryption Scheme with Provable Security. Inf. Process. Lett. 2011, 111, 661-666. [CrossRef] 
26. Lin, H.Y.; Wu, T.S.; Huang, S.K. An Efficient Strong Designated Verifier Proxy Signature Scheme for Electronic Commerce. J. Inf. Sci. Eng. 2012, 28, 771-785.

27. Lin, H.Y.; Wu, T.S.; Huang, T.Y.; Yeh, Y.S. Self-Certified Proxy Convertible Authenticated Encryption Scheme. In Proceedings of the 8th International Conference on Intelligent System Design and Applications (ISDA 2008), Kaohsiung, Taiwan, 26-28 November 2008; pp. 479-483.

28. Lu, C.F.; Hsu, C.L.; Lin, H.Y. Provably Convertible Multi-Authenticated Encryption Scheme for Generalized Group Communications. Inf. Sci. 2012, 199, 154-166. [CrossRef]

29. Wu, T.S.; Chen, Y.S.; Lin, H.Y.; Chang, T.K. Authenticated Encryption Scheme Based on Paillier System with Verifiable Public Keys. Commun. Comput. Secur. 2012, 2, 1-5. [CrossRef]

30. Wu, T.S.; Lin, H.Y. Efficient Self-Certified Proxy CAE Scheme and Its Variants. J. Syst. Softw. 2009, 82, 974-980. [CrossRef]

31. Wu, T.S.; Lin, H.Y.; Tsao, S.H.; Ting, P.Y. On the Construction of DL-Based Convertible Authenticated Encryption Scheme with Message Linkages. Inf. Int. Interdiscip. J. 2013, 16, 7983-7994.

32. ISO/IEC 9594-8. Information Technology_Open Systems Interconnection-The Directory: Public-Key and Attribute Certificate Frameworks; International Organization for Standardization: Geneva, Switzerland, 2001.

33. Pointcheval, D.; Stern, J. Security Arguments for Digital Signatures and Blind Signatures. J. Cryptol. 2000, 13, 361-369. [CrossRef]

34. Lee, J.S.; Chang, J.H.; Lee, D.H. Forgery Attacks on Kang et al.'s Identity-Based Strong Designated Verifier Signature Scheme and Its Improvement with Security Proof. Comput. Electr. Eng. 2010, 36, 948-954. [CrossRef]

35. Islam, S.K.H.; Biswas, G.P. Provably Secure Certificateless Strong Designated Verifier Signature Scheme Based on Elliptic Curve Bilinear Pairings. J. King Saud Univ.-Comput. Inf. Sci. 2013, 25, 51-61.

36. Cao, X.; Kou, W.; Du, X. A Pairing-Free Identity-Based Authenticated Key Agreement Protocol with Minimal Message Exchanges. Inf. Sci. 2010, 180, 2895-2903. [CrossRef]

(C) 2019 by the author. Licensee MDPI, Basel, Switzerland. This article is an open access article distributed under the terms and conditions of the Creative Commons Attribution (CC BY) license (http://creativecommons.org/licenses/by/4.0/). 